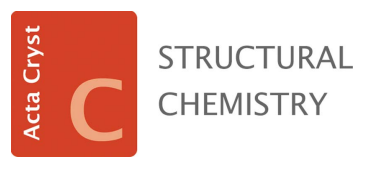

ISSN 2053-2296
Keywords: book review; inorganic chemistry; inorganic substances; Pauling file

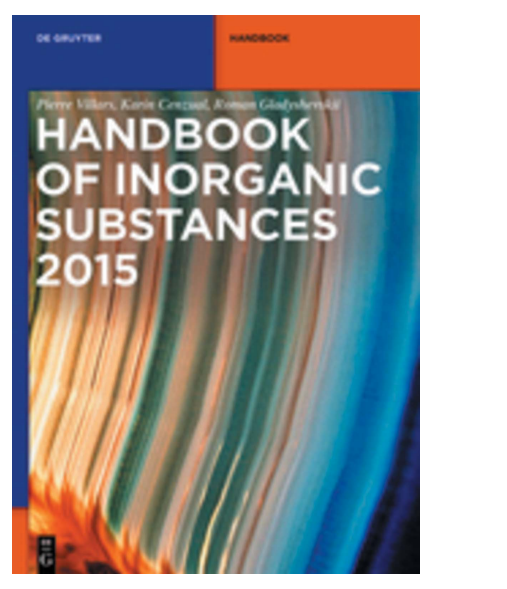

\section{Handbook of Inorganic Substances 2015. By Pierre Villars, Karin Cenzual \& Roman Gladyshevskii. De Gruyter, 2015. Hardcover, Pp. XIV+1801. Price EUR 249.00, USD 349.00, GBP 186.99. ISBN 978-3-11-031113-6.}

\author{
Pierrick Durand* \\ Université de Lorraine, CRM2, UMR 7036, Vandoeuvre-les-Nancy, F-54506, France, and CNRS, CRM2, UMR 7036, \\ Vandoeuvre-les-Nancy, F-54506, France. *Correspondence e-mail: pierrick.durand@univ-lorraine.fr
}

The aim of this handbook is to provide researchers and students with a comprehensive compilation of presently defined crystallographically identified inorganic substances within one volume. Owing to the explosion of crystallographic data published yearly, over 100000 inorganic structures are classified and presented within the massive handbook (over 1800 pages). The handbook is updated and refreshed yearly and this edition is the fourth release, the first having been released in 2011.

The preface is rather concise (3 pages) and describes clearly how to read and access the data within the handbook. The substances are sorted according to the number of chemical elements, then according to the alphabetical order of the elements and finally according to the chemical formula. It is relatively easy to search for and find inorganic substances in the handbook, and to obtain the bibliographic reference. Only minimal and vital information is given, viz. chemical formula/structure prototype/Hermann-Mauguin symbol for the space group/structural family, colour, density, a code indicating the level of structural studies and a handbook-specific reference number. Those wishing to find more information about structure prototypes or crystallographic vocabulary should visit the handbook website (http://paulingfile.com).

The Pauling file project started in 1995 and in that pre-internet time there is no doubt that such a handbook would have been a great help for anyone in the inorganic chemistry community looking for crystallographic information. Nowadays, collections of crystal structures of inorganic compounds are available online and are open access. There are, for example, currently more than 360000 entries in the Crystallography Open Database (COD; http://www.crystallography.net/cod/), and other online resources are also available. The handbook could be very useful in a wireless hotspot, otherwise I am not sure it will be able compete with other online and open-access resources.

In summary, the Handbook of Inorganic Substances 2015 is a valuable reference work for everyone working in the area of inorganic chemistry, and for students who wish to gain an insight into crystallographic aspects of this field. It will also provide stimulating ideas not only for chemists, but also for physicists and materials scientists. However, the editor should consider whether it is appropriate to release a new paper edition yearly or if it is not more appropriate to go online with open access and to provide not only cell parameters, but also the complete set of atomic parameters, such as is available in Crystallographic Information Files (CIFs). 2001 was not only the year of the space odyssey, it was also an important date for organic chemists and crystallographers as it saw the birth of a new journal, Acta Crystallographica Section E: Structure Reports Online, that revolutioned the compilation of crystallographic data for organic substances. It is now time for the online odyssey of the Handbook of Inorganic Substances to begin. 